\title{
Identification of IRF6 gene variants in three families with Van der Woude syndrome
}

\author{
ENE-CHOO TAN ${ }^{1}$, EILEEN CHEW-PING LIM ${ }^{1}$, SHIAO-HUI YAP ${ }^{3}$, SENG-TEIK LEE ${ }^{4}$, \\ JOANNE CHENG ${ }^{2}$, YONG-CHEN POR ${ }^{2}$ and VINCENT YEOW ${ }^{2}$
}

\begin{abstract}
${ }^{1}$ KK Research Centre, ${ }^{2}$ Cleft and Craniofacial Centre, KK Women's and Children's Hospital, 100 Bukit Timah Road, Singapore 229899; ${ }^{3}$ Population Genetics Programme, Defence Medical and Environmental Research Institute, DSO National Laboratories, 27 Medical Drive \#09-00, Singapore 117510; ${ }^{4}$ Department of Plastic Surgery, Singapore General Hospital, Outram Road, Singapore 169608, Singapore
\end{abstract}

Received January 8, 2008; Accepted February 20, 2008

\begin{abstract}
Van der Woude syndrome is the most common cause of syndromic orofacial clefting. It is characterised by the presence of lip pits, cleft lip and/or cleft palate. It is transmitted in an autosomal dominant manner, with high penetrance and variable expressivity. Several mutations in the interferon regulatory factor 6 (IRF6) gene have been found in VWS families, suggesting that this gene is the primary locus. We screened for mutations in this gene in three families in our population. There was a recurrent nonsense mutation within exon 9 of the gene for a Malay family consisting of five affected members with different presentations. We also found a co-segregating rare polymorphism which would result in a non-synonymous change 23 bases downstream of the nonsense mutation. This polymorphism was present in $<1 \%$ of the Malay subjects screened, but was not found among the Chinese and Indians in our population. For another family, a 396C $\rightarrow$ T mutation (R45W in the DNA-binding domain) was found in the proband, although the possibility of a genetic defect elsewhere could not be excluded because his mother and twin sister (both unaffected) also had this variant. In the third case with complete absence of family history, a de novo deletion spanning the whole IRF6 gene was detected in the child with VWS. This case of haploinsufficiency caused disruption of orofacial development but not other organ systems as the child has no other medical or developmental abnormalities despite the deletion of at least five other genes.
\end{abstract}

Correspondence to: Dr Ene-Choo Tan, KK Research Centre, KK Women's and Children's Hospital, 100 Bukit Timah Road, Singapore 229899, Singapore

E-mail: tanec@bigfoot.com

Key words: Van der Woude syndrome, interferon regulatory factor 6, direct sequencing, genetic variants, deletion

\section{Introduction}

Oral clefting is one of the most common congenital and craniofacial malformations in humans. The disfigurement of these patients can result in significant medical, surgical, and psychosocial complications. Infants with oral clefting may have secondary feeding, respiratory, speech, and auditory impairment. The incidence is at least 1 in 1,000 Caucasian live births but is higher in Singapore at 2.0, 1.7 and 2.07 per 1000 live births in three separate studies (1-3). Although significant advances have been made in the last few decades in terms of clinical management and treatment, multiple major surgical procedures and a lifetime of clinic visits are still required; in addition to emotional, physical, psychological and financial stress for the patient and their families.

Although over $>70 \%$ of cleft lip and/or cleft palate (CL/P) cases are non-syndromic, there are $>300$ syndromes which have cleft lip and/or cleft palate as one of the features (4-6). Among syndromic disorders, the highest number comprise cases of Van der Woude syndrome (VWS: OMIM 119300) which accounts for $2 \%$ of all cleft cases (6-9). Apart from the difference in the mode of inheritance which is autosomal dominant with high penetrance, its phenotype closely resembles the phenotype for the common non-syndromic forms of CL/P with complex pattern of inheritance (10). Other milder expressions of the phenotype include verrucous eminence in the lower lip, submucous cleft palate and bifid uvula.

Due to the similarity in the clefting phenotype, VWS could serve as a simpler model to study the more complex nonsyndromic forms of orofacial clefts. Despite being a Mendelian disorder, the high variability of expression even for family members carrying the same mutation is intriguing and suggests multifactorial influences similar to that of the non-syndromic clefts. In addition, the $15 \%$ of VWS with no lip pits are clinically indistinguishable from non-syndromic isolated clefts (11). Identification of mutations in this gene will offer more insight into the clinically significant changes in a population in which the incidence of cleft lip and palate is higher than global average. 


\section{Patients and methods}

Patient recruitment and clinical evaluation. Cases were recruited from the Department of Plastic, Reconstructive and Aesthetic Surgery of Singapore General Hospital and KK Women's and Children's Hospital. VWS diagnosis was established by the presence of lip pits in addition to orofacial clefting. Other data collected include father's medical history; the mother's medical and pregnancy history and exposure to common risk factors (including smoking and alcohol), a detailed family history including occurrence of all birth defects in relatives; and health and developmental assessment of the cases.

DNA samples. Ethical approval was obtained from the Institutional Review Board of Singapore General Hospital and KK Women's and Children's Hospital. Genomic DNA was extracted from blood in EDTA tubes or saliva which were collected from cases and immediate family members with informed consent.

Mutation screening. Genomic DNA was extracted using standard methods. Linkage analysis was carried out using PCR amplification for 7 microsatellite markers in the critical VWS1 region on 1q32-q41 (17). Screening of mutations in the IRF6 gene was carried out using PCR amplification with primers for exons and promoter region followed by direct sequencing with Big Dye Sequencing Kit (Applied Biosystems, Foster City, CA, USA). PCR products were analyzed by capillary electrophoresis on the ABI PRISM 3130 Genetic Analyzer (Applied Biosystems, Foster City, CA, USA). Sequence variants were verified on both strands and in all available family members.

SNP genotyping. A PCR-RFLP assay was developed for quick genotyping of control samples for the missense substitution in exon 9 found in Pedigree 1. Primers used are 5'- CCTCAG GGCCTCTTTGGTC-3' and 5'-CAATTTCAGGCACTACTC CAATC-3'. Amplification was performed in a $10 \mu 1$ reaction volume consisting of $10 \mathrm{mM}$ Tris- $\mathrm{HCl}, 1.5 \mathrm{mM} \mathrm{MgCl}, 50 \mathrm{mM}$ $\mathrm{KCl}, \mathrm{pH}$ 8.3, $0.2 \mathrm{mM}$ dNTPs, 5 pmoles of each primer, $0.5 \mathrm{U}$ of Taq polymerase and genomic DNA. Reaction conditions are initial denaturation at $95^{\circ} \mathrm{C}$ for $3 \mathrm{~min}, 35$ cycles of denaturation at $95^{\circ} \mathrm{C}$ for $30 \mathrm{sec}$, annealing at $60^{\circ} \mathrm{C}$ for $30 \mathrm{sec}$, extension at $72^{\circ} \mathrm{C}$ for $30 \mathrm{sec}$, and a final extension at $72^{\circ} \mathrm{C}$ for $5 \mathrm{~min}$. Amplicons were incubated with AciI according to manufacturer's recommendations (New England Biolabs). The restricted products were electrophoresed on a $2 \%$ agarose gel. For allele G, three bands of sizes 457, 179 and 63 bp are expected while allele A has only two bands of 457 and 242 bp.

For the novel third exon substitution $(396 \mathrm{C} \rightarrow \mathrm{T})$ found in Pedigree 2, a PCR-RFLP assay was developed for quick genotyping of control samples for the missense substitution. Primers used are 5'-GGCTAGAGCATGAAGTGTAA-3' and 5'-CATGCCCCCAAAAGAGGAAT-3'. Amplicons were incubated with HpaII according to manufacturer's recommendations (New England Biolabs). The restricted products were electrophoresed on a $1.5 \%$ agarose gel. For allele C, three bands of sizes 245, 204, 108 bp are expected while allele T has only two bands of 312 and $245 \mathrm{bp}$.
Copy number analysis. For multiplex ligation probe amplification analysis (MLPA), primers targeting the 1q32-q41 region were designed and the melting temperatures of the DNA-DNA hybrids determined by Raw Probe downloaded from MRC-Holland (http://www.mrc-holland.com/pages/ support_desing_synthetic_probespag.html). DNA (50 ng) from the proband with the suspected deletion and his family members was used in the MLPA protocol according to the manufacturer's instructions (MRC-Holland, Amsterdam, The Netherlands). PCR products $(2 \mu \mathrm{l})$ were analyzed by capillary electrophoresis on the ABI Prism 3130 Genetic Analyzer (Applied Biosystems, Foster City, CA, USA). Data analysis was performed using the Coffalyser software (www.mpla.com).

\section{Results}

Clinical findings. Three families were recruited into the study. The child from a Malay family (Pedigree 1) had strong family history with all three of her siblings affected. The parents and other siblings were also examined to ascertain the features are consistent with VWS before recruitment into the study. Clinical examination revealed that the proband and her father both had complete bilateral cleft of the lip and palate and two lower lip pits as did another child, a daughter aged 7 . The oldest sibling, a boy aged 11 presented with two lower lip pits only while the second oldest sibling, another boy aged 10 presented with a complete unilateral cleft of the left lip and palate and two lower lip pits.

The two other cases had unaffected parents and siblings. One was from Chinese family with a dizygotic twin pair discordant for both gender and VWS phenotype. The affected male had an affected maternal uncle (long deceased and genetic material unavailable) but his two parents were unaffected. The affected male had a complete right cleft lip and palate with lower lip pits. The proband from Pedigree 3 is a Chinese boy with no family history. He had complete bilateral cleft lip and palate and lower lip pits. He had two unaffected siblings, a younger brother and a younger sister. Besides the VWS facial features, there was no other clinically significant abnormality in other organ systems.

Pedigree 1. Direct sequencing of all nine exons was performed on the parents and all four affected children. There were two sequence changes in exon 9 , a $\mathrm{C} \rightarrow \mathrm{T}$ change at position 1234 which converted the arginine-coding codon to a stop codon, and a $\mathrm{G} \rightarrow \mathrm{A}$ change 23 nucleotides downstream which would translate into histidine in place of arginine (Fig. 1). No mutation was found in exons $3,4,7$ and 8 which were reported to have the most number of mutations. We found a polymorphism in near exon 4 and one in exon 7 , and one near exon 7 but these are not likely to be causative as they were also present in the general population.

To investigate whether the missense mutation might be a polymorphism, we genotyped 119 unrelated individuals from the same ethnic group. We found two heterozygotes, with the rest being homozygotes for the wild-type. Sequencing of these two samples confirmed that they had the missense substitution but not the nonsense mutations. We had no phenotypic information on the two control subjects but they were unlikely to be VWS cases since the syndrome is so rare. We 
A
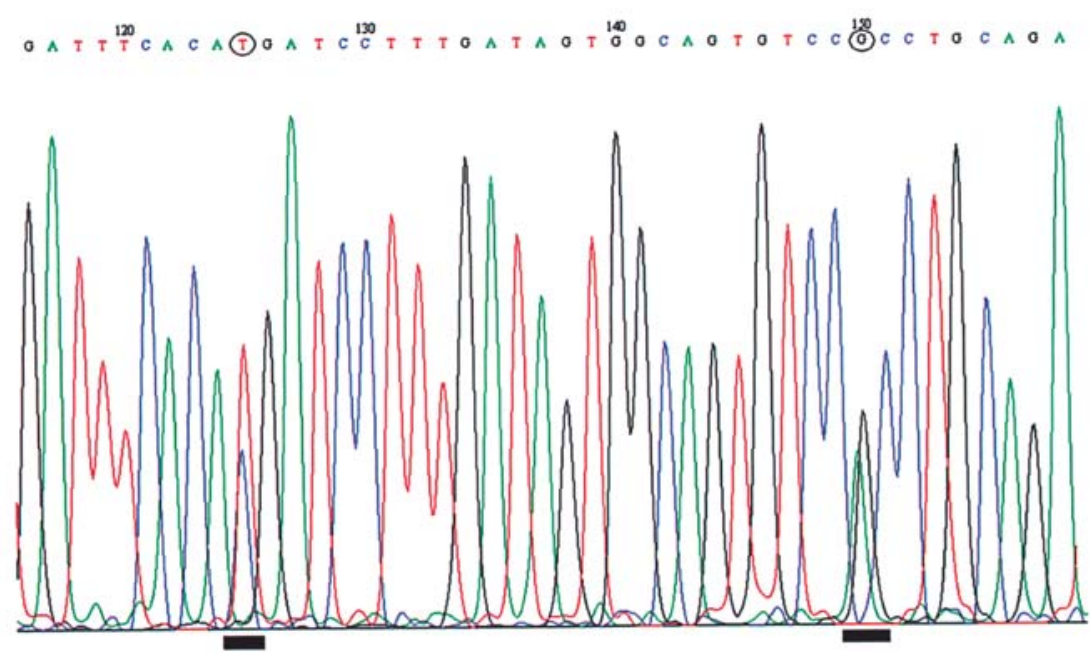

B

$$
\text { OATT TCA }
$$

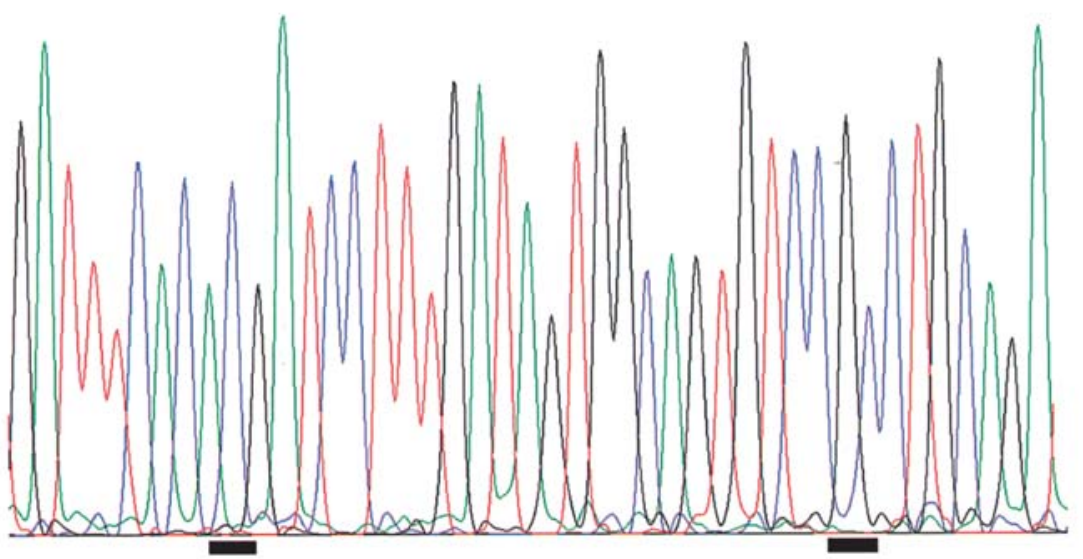

Figure 1. Chromatograms showing the two different mutations (underlined) identified in the proband with VWS with $1234 \mathrm{C} \rightarrow \mathrm{T}$ mutation and $1259 \mathrm{G} \rightarrow \mathrm{A}$ variant (A) and her unaffected mother carrying wild-type alleles at both positions (B).

A
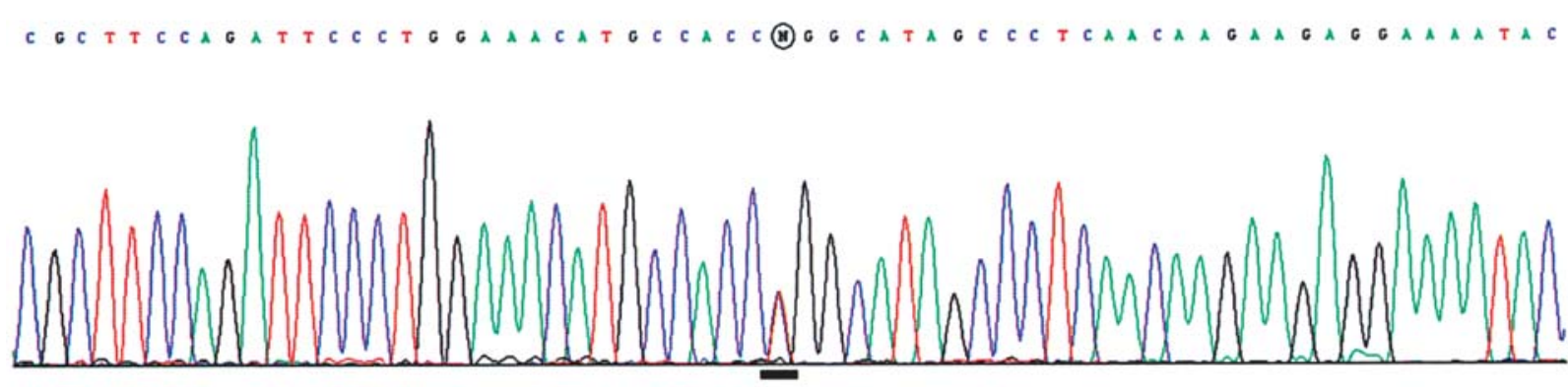

B

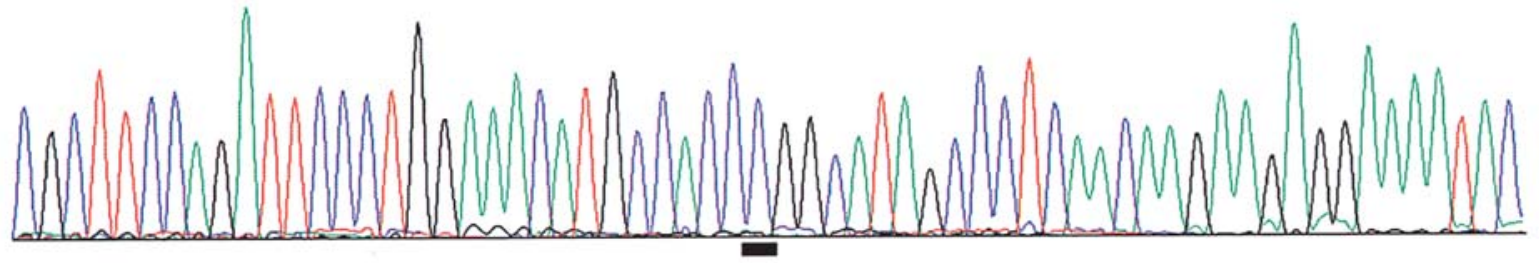

Figure 2. Chromatograms showing the 396C $\rightarrow$ T mutation (underlined) identified in the proband with VWS (A) and his unaffected mother carrying the wildtype alleles (B). 
Table I. Results from DNA sequencing showing the homozygous status of polymorphic markers in the proband for Pedigree 3 .

\begin{tabular}{lccccc}
\hline & Father & Mother & Proband & Sister & Brother \\
\hline rs3028138 & $-/-$ & CAAATA/CAAATA & CAAATA/CAAATA & CAAATA/- & CAAATA/- \\
rs1005287 & $\mathrm{T} / \mathrm{T}$ & $\mathrm{C} / \mathrm{C}$ & $\mathrm{C} / \mathrm{C}$ & $\mathrm{C} / \mathrm{T}$ & $\mathrm{C} / \mathrm{T}$ \\
rs2357229 & $\mathrm{A} / \mathrm{A}$ & $\mathrm{C} / \mathrm{C}$ & $\mathrm{C} / \mathrm{C}$ & $\mathrm{C} / \mathrm{A}$ & $\mathrm{C} / \mathrm{A}$ \\
rs6659549 & $\mathrm{C} / \mathrm{C}$ & $\mathrm{A} / \mathrm{A}$ & $\mathrm{A} / \mathrm{A}$ & $\mathrm{C} / \mathrm{A}$ & $\mathrm{C} / \mathrm{A}$ \\
rs7545542 & $\mathrm{A} / \mathrm{A}$ & $\mathrm{G} / \mathrm{G}$ & $\mathrm{G} / \mathrm{G}$ & $\mathrm{G} / \mathrm{A}$ & $\mathrm{G} / \mathrm{A}$ \\
rs7545538 & $\mathrm{C} / \mathrm{C}$ & $\mathrm{G} / \mathrm{G}$ & $\mathrm{G} / \mathrm{G}$ & $\mathrm{G} / \mathrm{C}$ & $\mathrm{G} / \mathrm{C}$ \\
rs2235377 & $\mathrm{A} / \mathrm{G}$ & $\mathrm{A} / \mathrm{A}$ & $\mathrm{A} / \mathrm{A}$ & $\mathrm{A} / \mathrm{A}$ & $\mathrm{A} / \mathrm{A}$ \\
rs7552506 & $\mathrm{C} / \mathrm{G}$ & $\mathrm{C} / \mathrm{C}$ & $\mathrm{C} / \mathrm{C}$ & $\mathrm{C} / \mathrm{G}$ & $\mathrm{C} / \mathrm{G}$ \\
rs17015224 & $\mathrm{C} / \mathrm{T}$ & $\mathrm{C} / \mathrm{C} / \mathrm{T}$ & $\mathrm{C} / \mathrm{T}$ \\
rs2013162 & $\mathrm{T} / \mathrm{T}$ & $\mathrm{G} / \mathrm{G}$ & $\mathrm{C} / \mathrm{C}$ & $\mathrm{G} / \mathrm{T}$ \\
rs2235375 & $\mathrm{G} / \mathrm{G}$ & $\mathrm{C} / \mathrm{C}$ & $\mathrm{G} / \mathrm{G}$ & $\mathrm{C} / \mathrm{G}$ & $\mathrm{C} / \mathrm{G}$ \\
rs2235371 & $\mathrm{G} / \mathrm{A}$ & $\mathrm{G} / \mathrm{G}$ & $\mathrm{C} / \mathrm{C}$ & $\mathrm{G} / \mathrm{G}$ & $\mathrm{G} / \mathrm{G}$ \\
rs2073485 & $\mathrm{T} / \mathrm{T}$ & $\mathrm{C} / \mathrm{C}$ & $\mathrm{G} / \mathrm{G}$ & $\mathrm{C} / \mathrm{T}$ & $\mathrm{C} / \mathrm{T}$ \\
rs742215 & $\mathrm{T} / \mathrm{T}$ & $\mathrm{A} / \mathrm{A}$ & $\mathrm{C} / \mathrm{C}$ & $\mathrm{A} / \mathrm{T}$ & $\mathrm{A} / \mathrm{T}$ \\
rs742214 & $\mathrm{G} / \mathrm{G}$ & $\mathrm{A} / \mathrm{A}$ & $\mathrm{A} / \mathrm{A}$ & $\mathrm{A} / \mathrm{G}$ & $\mathrm{A} / \mathrm{G}$ \\
rs2235372 & $\mathrm{T} / \mathrm{T}$ & $\mathrm{C} / \mathrm{C}$ & $\mathrm{A} / \mathrm{A}$ & $\mathrm{C} / \mathrm{T}$ & $\mathrm{C} / \mathrm{T}$ \\
rs680331 & $\mathrm{T} / \mathrm{T}$ & $\mathrm{C} / \mathrm{C}$ & $\mathrm{C} / \mathrm{C}$ & $\mathrm{C} / \mathrm{T}$ & $\mathrm{C} / \mathrm{T}$ \\
rs1044516 & $\mathrm{A} / \mathrm{A}$ & $\mathrm{C} / \mathrm{C}$ & $\mathrm{C} / \mathrm{C}$ & $\mathrm{A} / \mathrm{C}$ & $\mathrm{A} / \mathrm{C}$ \\
rs1856161 & $\mathrm{G} / \mathrm{A}$ & $\mathrm{G} / \mathrm{G}$ & $\mathrm{C} / \mathrm{C}$ & $\mathrm{G} / \mathrm{G}$ & $\mathrm{G} / \mathrm{G}$ \\
\hline
\end{tabular}

also genotyped 100 Chinese and 100 Indians from the local population but did not find any with the rare allele.

Pedigree 2. Microsatellite analysis and direct sequencing of all nine exons including the promoter region were performed on the genomic DNA from the proband and the parents. There was a $\mathrm{C} \rightarrow \mathrm{T}$ change in the $3 \mathrm{rd}$ exon or position 396 of the mRNA (NM_006147) which would result in a non-synonymous substitution of arginine by tryptophan (R45W) within the DNA-binding domain of the protein (Fig. 2). The proband was heterozygous for this variant which he inherited from his mother who was also heterozygous at this position. The sibling without the VWS phenotype was also heterozygous. Genotyping of 100 chromosomes in population control samples by PCR-RFLP showed that all were negative for this variant.

Pedigree 3. Sequencing of all nine exons and the promoter region revealed 21 polymorphisms but no potential causative mutation. However, it was observed that the patient was homozygous at all the polymorphic positions. He had not inherited any of the paternal alleles even when the father and mother were homozygous for different alleles at several positions. His two unaffected siblings were heterozygous at these positions and had clear inheritance of both paternal and maternal alleles (Table I).

MLPA analysis carried out confirmed the loss of copy number in the IRF6 and plexin 2 (Fig. 3). Analysis of ten additional markers within the region placed the deletion breakpoints at between complement component receptor 1 (CR1) and plexin A2 (PLXNA2) at the end nearer the

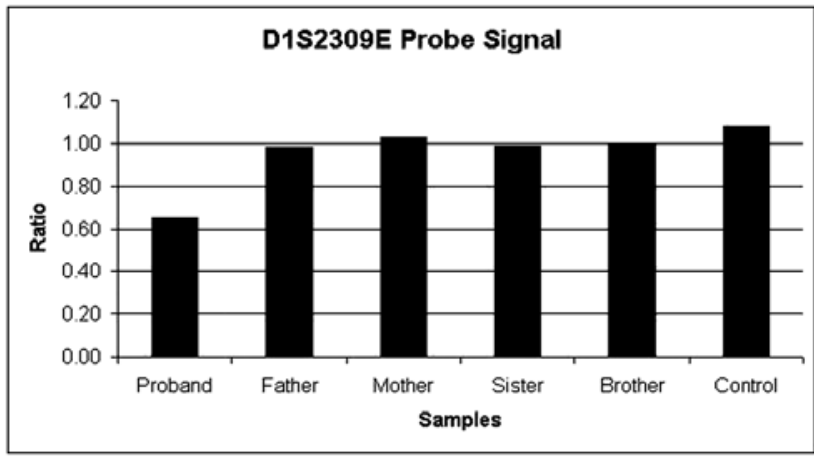

Figure 3. Results from MLPA analysis showing copy number difference.

centromere and between IRF6 and Synaptotagmin 14 (SYT14) at the telomeric end. The deletion is estimated to be between 1.7 and $3.0 \mathrm{Mb}$.

\section{Discussion}

Three chromosomal regions have been reported to be linked to VWS: 1p34, 1q32-41, and 17p11.2-11.1 (12-14). We used microsatellite markers specific for 1q32-41 because specific mutations in this region had been identified in many VWS families. As our result was consistent with the causative mutation on this region, we went on to screen the interferon regulatory factor gene (IRF6) which have shown positive linkage or association in other populations. 
The nonsense mutation we found in Pedigree 1 had been reported in another VWS family and was most likely the causative mutation. The family in this study is of Malay descent. These data are the first for mutation identified in the Malay population. For this family, at least four generations are affected with the grandmother and great grandfather of the index patient also having the phenotype. DNA was also obtained from two siblings of the affected father to confirm the segregation of the mutation with the VWS phenotype in this family. However, one of the reportedly unaffected sisters was found to have the mutation. As she was not available for clinical examination, it was possible that she might have more subtle submucous $\mathrm{CP}$ or there was incomplete penetrance of the phenotype. The other unaffected sibling had the wildtype sequence.

It has been reported that there is an apparent overtransmission of the valine allele to affected subjects for this common IRF6 polymorphism (15). In our pedigree, the affected father was heterozygous for this marker while the unaffected mother is homozygous for the valine allele. However, all the affected children received the isoleucine allele from the father.

For Pedigree 2, although the novel variant was also found in the unaffected parent and sibling, it could not be ruled out that it might predispose to VWS in the presence of additional genetic or environmental factor which was only encountered in the proband and his maternal uncle but not in the mother or sister, or that penetrance is higher in males. Additional work is also needed to investigate the effect of the change from a hydrophilic to an aromatic- hydrophobic amino acid residue on the function of the protein. There are records of seven twins with VWS in the literature, with four discordant pairs $(16,17)$. A similar scenario of a missense mutation (exon 2 250C $\rightarrow \mathrm{G}$ ) which segregated with two boys with VWS but was apparently non-penetrant in the mother has been reported (18).

For Pedigree 3, there was clear evidence that the paternal copy of the IRF6 gene was deleted in the patient. The de novo deletion spanned the entire IRF6 gene. There are at least four other documented cases which involve deletion (19-22). We are designing assays to map the size of the deletion and the exact breakpoints for this chromosomal region. It is estimated that at least five genes were deleted including calcium/calmodulin-dependent protein kinase $1 \mathrm{G}$ (CAMK1G), laminin beta 3 (LAMNB3) and hydroxysteroid (11-beta) dehydrogenase 1 (HSD11B1). Mapping of the boundary would determine which genes are disrupted and/or deleted and the consequence of having only one functional copy. As deletions are often mediated by sequences which are homologous or repetitive and are hotspots for recombination, it would also add to the knowledge of the characteristics of such sequences and the unequal recombination process.

In conclusion, the results reported here include a family with many affected members and clear autosomal dominant inheritance but presenting with mixed phenotype of either unilateral or bilateral cleft lip with cleft palate and lip pits only, an apparently male-only inheritance in another family, and a de novo deletion in a third family with no family history. Two new variants and a deletion were identified, adding to the spectrum of mutation for this gene/syndrome worldwide and also providing more cases for the study of correlation with expression of the phenotype and the pattern of variable penetrance.

\section{Acknowledgments}

This study was supported by SingHealth Grant no. EX057/ 2004 and KRAU 99/06 from the National Medical Research Council. We thank Drs Jeffrey C. Murray and Brian Schutte for primers and technical advice.

\section{References}

1. Fong PH, Yeap CL and Lee ST: Congenital cleft lip and palate in Singapore. Ann Acad Med Singapore 12: 363-365, 1983.

2. Tan KL: Incidence and epidemiology of cleft lip/palate in Singapore. Ann Acad Med Singapore 17: 311-314, 1988.

3. Yi NN, Yeow VK and Lee ST: Epidemiology of cleft lip and palate in Singapore - a 10-year hospital-based study. Ann Acad Med Singapore 28: 655-659, 1999.

4. Jones MC: Etiology of facial clefts: prospective evaluation of 428 patients. Cleft Palate J 25: 16-20, 1988.

5. Murray JC: Gene/environment causes of cleft lip and/or palate. Clin Genet 61: 248-256, 2002.

6. Schutte BC and Murray JC: The many faces and factors of orofacial clefts. Hum Mol Genet 8: 1853-1859, 1999.

7. Rizos $\mathrm{M}$ and Spyropoulos MN: Van der Woude syndrome: a review. Cardinal signs, epidemiology, associated features, differential diagnosis, expressivity, genetic counselling and treatment. Eur J Orthod 26: 17-24, 2004.

8. Rintala AE and Ranta R: Lower lip sinuses: I. Epidemiology, microforms and transverse sulci. Br J Plast Surg 34: 26-30, 1981.

9. Burdick AB: Genetic epidemiology and control of genetic expression in van der Woude syndrome. J Craniofac Genet Dev Biol (Suppl) 2: 99-105, 1986.

10. Van Der Woude A: Fistula labii inferioris congenita and its association with cleft lip and palate. Am J Hum Genet 6: 244-256, 1954.

11. Burdick AB, Bixler D and Puckett CL: Genetic analysis in families with van der Woude syndrome. J Craniofac Genet Dev Biol 5: 181-208, 1985.

12. Koillinen H, Wong FK, Rautio J, et al: Mapping of the second locus for the Van der Woude syndrome to chromosome 1p34. Eur J Hum Genet 9: 747-752, 2001.

13. Schutte BC, Bjork BC, Coppage KB, et al: A preliminary gene map for the Van der Woude syndrome critical region derived from $900 \mathrm{~kb}$ of genomic sequence at 1q32-q41. Genome Res 10: 81-94, 2000

14. Sertie AL, Sousa AV, Steman S, Pavanello RC and PassosBueno MR: Linkage analysis in a large Brazilian family with van der Woude syndrome suggests the existence of a susceptibility locus for cleft palate at 17p11.2-11.1. Am J Hum Genet 65: 433-440, 1999.

15. Zucchero TM, Cooper ME, Maher BS, et al: Interferon regulatory factor 6 (IRF6) gene variants and the risk of isolated cleft lip or palate. N Engl J Med 351: 769-780, 2004.

16. Tokat C, Bilkay U, Songur E and Akin Y: Van der Woude syndrome in twins. J Craniofac Surg 16: 936-939, 2005.

17. Kondo S, Schutte BC, Richardson RJ, et al: Mutations in IRF6 cause Van der Woude and popliteal pterygium syndromes. Nat Genet 32: 285-289, 2002.

18. Item CB, Turhani D, Thurnher D, et al: Van Der Woude syndrome: variable penetrance of a novel mutation (p.Arg $84 \mathrm{Gly}$ ) of the IRF6 gene in a Turkish family. Int J Mol Med 15: 247-251, 2005.

19. Bocian M and Walker AP: Lip pits and deletion 1q32-41. Am J Med Genet 26: 437-443, 1987.

20. Sander A, Schmelzle R and Murray J: Evidence for a microdeletion in 1q32-41 involving the gene responsible for Van der Woude syndrome. Hum Mol Genet 3: 575-578, 1994.

21. Schutte BC, Basart AM, Watanabe Y, et al: Microdeletions at chromosome bands 1q32-q41 as a cause of Van der Woude syndrome. Am J Med Genet 84: 145-150, 1999.

22. Kayano S, Kure S, Suzuki Y, et al: Novel IRF6 mutations in Japanese patients with Van der Woude syndrome: two missense mutations (R45Q and P396S) and a 17-kb deletion. J Hum Genet 48: 622-628, 2003. 